\title{
From the Desk of THE EdiTOR-IN-CHIEF
}

\section{What Ails Postgraduate Medical Education in India? N.Ananthakrishnan *}

The fact that postgraduate medical education in India is far from ideal or for that matter even satisfactory is well known to all those involved in the field. In reality it is on a rapid downhill course which in a few years is likely to cause disastrous consequences to the health of the country. Surprisingly little action is forthcoming from any quarter to set matters right. What is happening is only tinkering at the periphery of the issues without addressing them headlong and trying to find a solution. Some of the glaring deficiencies in the system are worth being mentioned herein. It is impossible, however, in one presentation to cover all that requires attention. In the absence of any fixed norms as entry criteria or a national standard, the quality of postgraduates in any program varies widely from institution to institution depending on the admission process. This makes standardization of the training near impossible and attainment of uniform goals near non-achievable. There is total lack of clarity of what differentiates a diploma course from a degree course other than the duration and the requirement of a dissertation for the latter. The syllabus is more or less the same, the teaching learning activities are more or less parallel and often common and the examination in many instances is not different either in the pattern or the degree of difficulty or the manner of assessment. The diploma course, therefore, has become the refuge of the destitute who do not make it or can't afford to make it to the degree course and is used as a preparatory phase while waiting to join the degree course at some future period. Originally the diploma was meant as an end qualification to enable doctors to be trained sufficiently to practice as specialists in secondary and primary care areas. It may be better in the light of loss of popularity of the diploma course to abolish it altogether.

The curriculum of the postgraduate courses has not been revised for decades. Archaic contents have not been deleted nor new contents added with any systematicity. This is sought to be obfuscated by saying that one of the papers should be on recent advances. No one knows what is "recent" and what is meant by "advances." The number of seats is fixed in somewhat of an arbitrary matter and is subject to an annual increase when it should be re-fixed only when the previous batch of increased number of students completes the course. This is the procedure adopted for undergraduates. Also since eight units have been made mandatory for 250 undergraduate admissions in subjects likely surgery and medicine, linking postgraduate seats to units (say maximum of two per unit) would be better than linking them to number of teachers eligible to be postgraduate teachers. In the latter case far more number of postgraduates are likely to be admitted than the clinical load warrants thus leaving serious deficiencies in training. A serious deficiency is lack of a standard subject specific logbook for monitoring postgraduate training. This is sought to be done to some extent in the courses conducted by the National Board but is absent in the MCI system. Even if adopted in some institutions it finds no place or no weightage in the evaluation process. Surprisingly, in a skilloriented profession like medicine, there is no importance at all given in postgraduate education for continuous internal assessment. It is well known that proper assessment of a postgraduate as regards competency cannot be made in a brief encounter which is what the final examination is. All other professional courses have some weight assigned to internal assessment but not medicine. Why this is so remains obscure? A portfolio would be one method of addressing this issue but unless it becomes mandatory and advised bythe regulator it is not likely be universally adopted. The quality of training in research methodology and biostatistics is abysmal and extends to being non-existent in many institutions. As a result the quality of dissertations is very poor. Since the evaluation system of dissertations is hazy and lacks objectivity in many universities this state continues to persist. The process of objectivising dissertation evaluation has begun in some institutions a decade or more ago but is yet to catch on, once again since there is no mandatory regulation in this regard.

Finally one is forced to mention the archaic examination system which has not changed at all in substantial measure over fifty years or more. It remains more or less largely subjective, norm-referenced and without fixed list of competencies which need to be certified after ensuring their attainment. A postgraduate medical evaluation system should be competency based and criterion referenced. When this would happen remains obscure? Perhaps the stimulus for change has to come from bottom up - from the society, from the outgoing postgraduates who realise their deficiencies when faced with real life situations, from professional associations and from educationists. Surely it has to happen soon. This issue of annals deals with some of the recent developments in the field of medical education pertinent to this matter.

\footnotetext{
* N. Ananthakrishnan, Dean, Research and Postgraduate Studies, SBV

Professor of Surgery,

MGMCRI (SBV), Pondicherry
} 